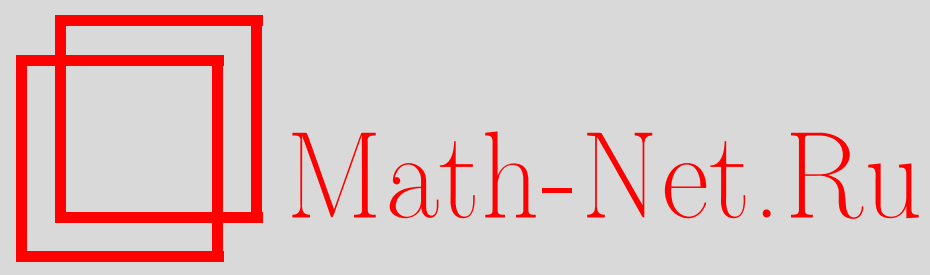

Е. И. Бережной, Теорема исправимости для анизотропных пространств, Матем. заметки, 2001, том 70, выпуск 3, 323333

DOI: https://doi.org/10.4213/mzm745

Использование Общероссийского математического портала Math-Net.Ru подразумевает, что вы прочитали и согласны с пользовательским соглашением http://www . mathnet.ru/rus/agreement

Параметры загрузки:

IP : 54.172 .240 .79

26 апреля 2023 г., 16:29:30

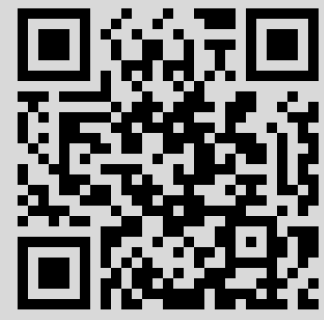




\title{
ТЕОРЕМА ИСПРАВИМОСТИ \\ ДЛЯ АНИЗОТРОПНЫХ ПРОСТРАНСТВ
}

\section{Е.И. Бережной}

\begin{abstract}
В работе решена следующая старая задача. Пусть фиксировано $\varepsilon>0$ и функция $f:[0,1]^{n} \rightarrow \mathbb{R}$ с заданными частными модулями непрерьвности, вычисленньми в симметричном пространстве $X$. Требуется указать множество $I(\varepsilon)$ с мерой больше $1-\varepsilon$ такое, что частные равномерные модули непрерьвности функции $f$, вычисленные по точкам этого множества, допускают неулучшаемую (относительно всех сужений на множества с мерой больше $1-\varepsilon$ ) оценку частных равномерных модулей непрерывности, и вьписать эту оценку равномерных частных модулей непрерывности.

Библиография: 9 названий.
\end{abstract}

Известную теорему Н.Н. Лузина о $C$-свойстве измеримой функции $f: I^{n} \rightarrow \mathbb{R}$ можно переформулировать следуюшим образом: для всякого $\varepsilon>0$ найдется множество $I(\varepsilon) \mathrm{c}$ мерой больше $1-\varepsilon$ такое, что равномерньй модуль непрерьвности функции $f$, вычисленный по точкам этого множества, стремится к нулю. В дальнейшем теорема Лузина развивалась по двум руслам: одно из них - это теоремы исправимости, т.е. нахождение функции $g$ с хорошими свойствами, для которой $\mu(\{t: g(t) \neq f(t)\})<1-\varepsilon$, а второе оценки некоторых характеристик функции $f$ на множествах меры больше $1-\varepsilon$. K теоремам первого направления можно отнести теоремы типа Д. Меньшова [1], к теоремам второго типа относятся, в основном, многомерные теоремы [2]-[4]. Это связано с тем, что теоремы первого типа связаны с теоремами второго типа через теорему о продолжении, а теоремы о продолжении в $\mathbb{R}^{n}$ существенно сложнее одномерных.

Настояшая работа посвящена решению следуюшей старой задачи. Пусть фиксировано $\varepsilon>0$ и функция $f: I^{n} \rightarrow \mathbb{R}$ с заданными частными модулями непрерьвности, вычисленными в симметричном пространстве $X$. Требуется указать множество $I(\varepsilon)$ с мерой больше $1-\varepsilon$ такое, что частные равномерные модули непрерьвности функции $f$, вычисленные по точкам этого множества, допускают неулучшаемую (относительно всех сужений на множества с мерой больше $1-\varepsilon$ ) оценку частных равномерных модулей непрерьвности, и вьписать эту оценку частных равномерных модулей непрерывности. Полньй ответ для этой задачи для пространства $L^{p}$ в одномерном случае дал К. Осколков [5], [6]. В многомерном случае для изотропного случая эта задача разобрана автором [2]. Отметим, что ранее для изотропного случая некоторые многомерные результаты для $L^{p}$ были получены Ю. Брудным и В. Колядой [3], [4].

Работа выполнена при частичной поддержке Российского фонда фундаментальных исследований, грант № 99-01-00355. 
Схема решения основной задачи достаточно традиционна для теорем исправимости. Сначала данная функция представляется в виде суммы ряда $\sum_{i=1}^{\infty} f_{i}$ гладких функций. Далее для каждой функции $f_{i}$ рассматривается ее сужение на множество большой меры $I_{i}$ такое, что на этом множестве функция имеет гарантированную гладкость. После этого искомое множество $I(\varepsilon)$ берется как пересечение множеств $I_{i}$.

Основной результат работы представлен в теоремах 1 и 2. Отметим, что если частные модули непрерьвности по всем направлениям совпадают, т.е. случай изотропный, основные результаты работы просто совпадают с основньми результатами работы автора [2]. Уместно заметить, что оценки равномерных модулей непрерьвности $f$ на $I(\varepsilon)$ зависят только от фундаментальной функции симметричного пространства, в котором вычисляются исходные модули непрерывности, и одинаковы для всех симметричных пространств с данной фундаментальной функцией. В конце работы показано, что основная теорема не может быть усилена.

Пусть $D$ - измеримое множество, через $\chi(D)$ обозначим его характеристическую функцию, а через $\mu(D)$ - его меру Лебега. Пусть $I^{n}$ - единичньй куб в $\mathbb{R}^{n}$ с обычной мерой Лебега, $S(\mu)$ - множество измеримых функций на $I^{n}$. Для $f: I^{n} \rightarrow \mathbb{R}$, как обычно, через $\lambda(f, \alpha)$ обозначим функцию распределения функции $f$, т.е. $\lambda(f, \alpha)=\mu(\{x$ : $|f(x)|>\alpha\}), \alpha \in \mathbb{R}_{+}$. Напомним [7], что банахово пространство функций назьвается симметричным, если из справедливости при всех $\alpha \in \mathbb{R}_{+}$неравенства $\lambda(g, \alpha) \leqslant \lambda(f, \alpha)$ и $f \in X$ следует, что $g \in X$ и $\|g|X\|\leqslant\| f| X\|$ (символом $\|f \mid X\|$ обозначается норма элемента $f$ в пространстве $X$ ). Важную информацию о симметричном пространстве $X$ несет его фундаментальная функция, которая определяется равенством $\psi(X, t)=$ $\|\chi(D) \mid X\|$, где $t=\mu(D)$.

Примерами симметричных пространств являются пространства Лебега $L^{p}(p \geqslant 1)$, пространства Орлича $L_{h}$, построенные по вьпуклой функции $h$, пространства Лоренца $\Lambda(\varphi)$ и Марцинкевича $M(\varphi)$, построенные по вогнутой функции $(\varphi)$. Для фундаментальных функций этих пространств справедливы равенства

$$
\psi\left(L^{p}, t\right)=t^{1 / p}, \quad \psi\left(L_{h}, t\right)=\frac{1}{h^{-1}\left(t^{-1}\right)}, \quad \psi(\Lambda(\varphi), t)=\psi(M(\varphi), t)=\varphi(t) .
$$

Ниже нам удобно считать, что каждая функция $f: I^{n} \rightarrow \mathbb{R}$ определена не только на $I^{n}$, но и доопределена на все $\mathbb{R}^{n}$ нулем.

Пусть $X$ - симметричное пространство, $e^{j}$ - единичный координатньй вектор в $\mathbb{R}^{n}$. Для каждой $f: I^{n} \rightarrow \mathbb{R}$ определим частный модуль непрерывности по переменному $x_{j}:$

$$
\omega_{j}(h, f, X)=\sup _{0<\delta \leqslant h}\left\|f\left(x+\delta e^{j}\right)-f(x) \mid X\right\| .
$$

Как обычно, через $\omega_{j}^{*}(h, f, X)$ обозначим наименьшую вогнутую мажоранту функции $\omega_{j}(h, f, X)$. Хорошо известно неравенство С. Б. Стечкина (см., например, [8, с. 154])

$$
\omega_{j}(h, f, X) \leqslant \omega_{j}^{*}(h, f, X) \leqslant 2 \omega_{j}(h, f, X) .
$$

Поскольку в дальнейших построениях величина абсолютных констант не играет особой роли, можно считать, что $\omega_{j}(h, f, X)$ - вогнутая функция.

Переходим к решению основной задачи. Для наглядности мы ограничимся случаем $n=2$. При других $n$ рассмотрения проходят аналогично. 
Сначала нам потребуются некоторыепростые утверждения из теории симметричных пространств [2], [7]. Пусть $X$ - симметричное пространство, $f \in X$ и задано $\alpha>0$. Положим $D(f, \alpha)=\{x:|f(x)|>\alpha\}$. Тогда справедливо неравенство

$$
\psi(X, \mu(D(f, \alpha))) \leqslant \frac{\|f \mid X\|}{\alpha} .
$$

Пусть функция $f: I^{2} \rightarrow \mathbb{R}$ имеет частные производные по первой и второй координатам. Введем обозначения

$$
f_{1}(x, y)=\frac{\partial}{\partial x} f(x, y), \quad f_{2}(x, y)=\frac{\partial}{\partial y} f(x, y) .
$$

Нам потребуется также следующий факт [2, лемма 6].

Лемма А. Пусть $X$ - симметричное пространство. Пусть $f: I^{2} \rightarrow \mathbb{R}, f_{1}(x, y)$ $\in X u f_{1}(x, y)$ - непрерывная на $I^{2}$ функция. Пусть $\gamma \in \mathbb{R}_{+}$фиксировано. Тогда существует мнохество $U_{1}(\gamma)$ такое, что выполнены условия:

а) если $\left(x_{1}, y\right) \in I^{2} \backslash U_{1}(\gamma)$, то для любой точки вида $\left(x_{2}, y\right) \in I^{2}$ верно неравенство

$$
\left|f_{1}\left(x_{1}, y\right)-f_{1}\left(x_{2}, y\right)\right| \leqslant 2 \gamma\left|x_{1}-x_{2}\right|
$$

б) для меры множества $U_{1}(\gamma)$ верно неравенство

$$
\psi\left(X, \mu\left(U_{1}(\gamma)\right)\right) \leqslant c_{0} \frac{\left\|f_{1} \mid X\right\|}{\gamma}
$$

(константа с сависит только от размерности, для $n=2$ можно положить $\left.c_{0}=25\right)$.

Аналогичные факты справедливы и в случае, когда известно, что $f_{2}(x, y) \in X$ и $f_{2}(x, y)$ непрерьвная на $I^{2}$ функция.

Для $h \in(0,1), \delta \in(0,1)$ и $f \in L^{1}$ положим

$$
f(h, \delta ; x, y)=\frac{1}{h} \frac{1}{\delta} \int_{0}^{h} \int_{0}^{\delta} f(x+t, y+s) d t d s .
$$

Из соотношения $\|f(h, \delta ; x, y)-f(x, y) \mid X\| \leqslant \omega_{1}(h, f, X)+\omega_{2}(\delta, f, X)$ сразу следует неравенство

$$
\begin{aligned}
& \left\|f\left(h_{1}, \delta_{1} ; x, y\right)-f\left(h_{2}, \delta_{2} ; x, y\right) \mid X\right\| \\
& \quad \leqslant \omega_{1}\left(h_{1}, f, X\right)+\omega_{1}\left(h_{2}, f, X\right)+\omega_{2}\left(\delta_{1}, f, X\right)+\omega_{2}\left(\delta_{2}, f, X\right) .
\end{aligned}
$$

Кроме того, верњы и неравенства

$$
\left\|f_{1}(h, \delta ; x, y)\left|X\left\|\leqslant \frac{\omega_{1}(h, f, X)}{h}, \quad\right\| f_{2}(h, \delta ; x, y)\right| X\right\| \leqslant \frac{\omega_{2}(\delta, f, X)}{\delta} .
$$

Напомним, что

$$
f_{1}(h, \delta ; x, y)=\frac{\partial}{\partial x} f(h, \delta ; x, y), \quad f_{2}(h, \delta ; x, y)=\frac{\partial}{\partial y} f(h, \delta ; x, y) .
$$


Теперь мы переходим к основной теореме. Зафиксируем $\varepsilon>0$, симметричное пространство $X$, непрерьвную функцию $f \in X$ с заданными частными модулями непрерьвности $\omega_{1}(h, f, X)$ и $\omega_{2}(\delta, f, X)$. Выберем теперь три монотонных последовательности положительных чисел $\left\{h_{i}\right\},\left\{\delta_{i}\right\}$ и $\left\{\varepsilon_{i}\right\}$ так, чтобы выполнялись условия

$$
\begin{gathered}
\lim _{i \rightarrow \infty} h_{i}=\lim _{i \rightarrow \infty} \delta_{i}=0, \\
\sum_{i=1}^{\infty} \varepsilon_{i}<\varepsilon
\end{gathered}
$$

причем выбор $\left\{h_{i}\right\},\left\{\delta_{i}\right\}$ и $\left\{\varepsilon_{i}\right\}$ будем осуществлять так, чтобы наряду с $(4)$ и (5) вьполнялось соотношение

$$
\sum_{i=1}^{\infty} \frac{\omega_{1}\left(h_{i}, f, X\right)+\omega_{2}\left(\delta_{i}, f, X\right)}{\psi\left(X ; \varepsilon_{i}\right)}<\infty .
$$

Условие (6) означает, что $\omega_{1}\left(h_{i}, f, X\right)$ и $\omega_{2}\left(\delta_{i}, f, X\right)$ достаточно быстро, а $\psi\left(X ; \varepsilon_{i}\right)$ достаточно медленно стремятся к нулю (напомним, что $\psi(X ; t)$ есть фундаментальная функция пространства $X)$. С последовательностями $\left\{h_{i}\right\},\left\{\delta_{i}\right\}$ и $\left\{\varepsilon_{i}\right\}$ и частными модулями непрерьвности $\omega_{1}\left(h_{i}, f, X\right)$ и $\omega_{2}\left(\delta_{i}, f, X\right)$ свяжем новые функции

$$
\begin{aligned}
& \Omega_{1}(h, f, X)=\inf _{k}\left\{h \sum_{i=1}^{k} \frac{\omega_{1}\left(h_{i}, f, X\right)}{h_{i} \psi\left(X ; \varepsilon_{i}\right)}+\sum_{i=k+1}^{\infty} \frac{\omega_{1}\left(h_{i-1}, f, X\right)+\omega_{2}\left(\delta_{i-1}, f, X\right)}{\psi\left(X ; \varepsilon_{i}\right)}\right\}, \\
& \Omega_{2}(\delta, f, X)=\inf _{k}\left\{\delta \sum_{i=1}^{k} \frac{\omega_{2}\left(\delta_{i}, f, X\right)}{\delta_{i} \psi\left(X ; \varepsilon_{i}\right)}+\sum_{i=k+1}^{\infty} \frac{\omega_{1}\left(h_{i-1}, f, X\right)+\omega_{2}\left(\delta_{i-1}, f, X\right)}{\psi\left(X ; \varepsilon_{i}\right)}\right\} .
\end{aligned}
$$

Эти функции являются вогнутыми, так как являются нижними гранями линейных функций и, кроме того, в силу (6) в нуле равны нулю.

Теорема 1. Пусть $X$ - симметричное пространство и его фундаментальная функиия $\psi(X, t)$ строго монотонна. Пусть задана непрерывная функиия $f(x, y)$ с заданными частными модулями непрерывности $\omega_{1}(h, f, X)$ u $\omega_{2}(\delta, f, X)$. Пусть задано $\varepsilon>0$ и пусть последовательности $\left\{h_{i}\right\},\left\{\delta_{i}\right\}$ u $\left\{\varepsilon_{i}\right\}$ удовлетворяют (4)-(6). Тогда существует мнохество $W(\varepsilon)$ такое, что выполнены соотношения:

a) $\mu(W(\varepsilon))<\varepsilon$;

б) если $\left(x_{1}, y\right),\left(x_{2}, y\right) \in I^{2} \backslash W(\varepsilon)$, mo

$$
\left|f\left(x_{1}, y\right)-f\left(x_{2}, y\right)\right| \leqslant\left|f\left(h_{0}, \delta_{0} ; x_{1}, y\right)-f\left(h_{0}, \delta_{0} ; x_{2}, y\right)\right|+c_{1} \Omega_{1}\left(\left|x_{1}-x_{2}\right|, f, X\right)
$$

в) $е с л и\left(x, y_{1}\right),\left(x, y_{2}\right) \in I^{2} \backslash W(\varepsilon), m o$

$$
\left|f\left(x, y_{1}\right)-f\left(x, y_{2}\right)\right| \leqslant\left|f\left(h_{0}, \delta_{0} ; x, y_{1}\right)-f\left(h_{0}, \delta_{0} ; x, y_{2}\right)\right|+c_{1} \Omega_{2}\left(\left|y_{1}-y_{2}\right|, f, X\right) .
$$

Здесь $c_{1}-$ абсолютная константа. Можно положить $c_{1}=\max \left\{16 c_{0}, 8\right\}$. 
ДокАЗАТЕЛЬСтво. Выберем числа $\alpha_{i}, \beta_{i}$ и $\gamma_{i}$ так, чтобы выполнялись соотношения

$$
\begin{aligned}
& \frac{\psi\left(X ; \varepsilon_{i}\right)}{2}=\frac{1}{\gamma_{i}}\left\{\omega_{1}\left(h_{i}, f, X\right)+\omega_{1}\left(h_{i-1}, f, X\right)+\omega_{2}\left(\delta_{i}, f, X\right)+\omega_{2}\left(\delta_{i-1}, f, X\right)\right\}, \\
& \frac{\psi\left(X ; \varepsilon_{i}\right)}{4}=2 \frac{c_{0}}{\alpha_{i}}\left\{\frac{\omega_{1}\left(h_{i}, f, X\right)}{h_{i}}+\frac{\omega_{1}\left(h_{i-1}, f, X\right)}{h_{i-1}}\right\} \\
& \frac{\psi\left(X ; \varepsilon_{i}\right)}{4}=2 \frac{c_{0}}{\beta_{i}}\left\{\frac{\omega_{2}\left(\delta_{i}, f, X\right)}{\delta_{i}}+\frac{\omega_{2}\left(\delta_{i-1}, f, X\right)}{\delta_{i-1}}\right\} .
\end{aligned}
$$

Положим

$$
\begin{gathered}
g_{i}(x, y)=f\left(h_{i}, \delta_{i} ; x, y\right)-f\left(h_{i-1}, \delta_{i-1} ; x, y\right), \\
g_{i, 1}(x, y)=\frac{\partial}{\partial x} g_{i}(x, y), \quad g_{i, 2}(x, y)=\frac{\partial}{\partial y} g_{i}(x, y) .
\end{gathered}
$$

Функции $g_{i}(x, y), g_{i, 1}(x, y), g_{i, 2}(x, y)$ непрерывны и для них согласно $(2),(3)$ верны оценки

$$
\begin{aligned}
\left\|g_{i}(x, y) \mid X\right\| & \leqslant \omega_{1}\left(h_{i-1}, f, X\right)+\omega_{1}\left(h_{i}, f, X\right)+\omega_{2}\left(\delta_{i-1}, f, X\right)+\omega_{2}\left(\delta_{i}, f, X\right), \\
\left\|g_{i, 1}(x, y) \mid X\right\| & \leqslant \frac{\omega_{1}\left(h_{i}, f, X\right)}{h_{i}}+\frac{\omega_{1}\left(h_{i-1}, f, X\right)}{h_{i-1}} \\
\left\|g_{i, 2}(x, y) \mid X\right\| & \leqslant \frac{\omega_{2}\left(\delta_{i}, f, X\right)}{\delta_{i}}+\frac{\omega_{2}\left(\delta_{i-1}, f, X\right)}{\delta_{i-1}} .
\end{aligned}
$$

Из (6) и последних соотношений следует, что справедливо равенство (ряд сходится в $X$ )

$$
f(x, y)=f\left(h_{0}, \delta_{0} ; x, y\right)+\sum_{i=1}^{\infty}\left\{f\left(h_{i}, \delta_{i} ; x, y\right)-f\left(h_{i-1}, \delta_{i-1} ; x, y\right)\right\}
$$

Для каждого $i$ для функций $g_{i}(x, y)$ и $g_{i, 1}(x, y)$ применим лемму А, полагая $\gamma=\alpha_{i} / 2$, a для функций $g_{i}(x, y)$ и $g_{i, 2}(x, y)$ применим лемму $\mathrm{A}$, полагая $\gamma=\beta_{i} / 2$. Тогда получим множества $U_{i, 1}$ и $U_{i, 2}$, удовлетворяюшие неравенствам

$$
\begin{aligned}
& \psi\left(X, \mu\left(U_{i, 1}\right)\right) \leqslant 2 \frac{c_{0}}{\alpha_{i}}\left\|g_{i, 1}(x, y) \mid X\right\| \leqslant 2 \frac{c_{0}}{\alpha_{i}}\left\{\frac{\omega_{1}\left(h_{i}, f, X\right)}{h_{i}}+\frac{\omega_{1}\left(h_{i-1}, f, X\right)}{h_{i-1}}\right\}, \\
& \psi\left(X, \mu\left(U_{i, 2}\right)\right) \leqslant 2 \frac{c_{0}}{\beta_{i}}\left\|g_{i, 2}(x, y) \mid X\right\| \leqslant 2 \frac{c_{0}}{\beta_{i}}\left\{\frac{\omega_{2}\left(\delta_{i}, f, X\right)}{\delta_{i}}+\frac{\omega_{2}\left(\delta_{i-1}, f, X\right)}{\delta_{i-1}}\right\}
\end{aligned}
$$

и, следовательно, согласно (12), (13) неравенству

$$
\begin{aligned}
\psi\left(X, \mu\left(U_{i, 1} \cup U_{i, 2}\right)\right) & \leqslant \psi\left(X, \mu\left(U_{i, 1}\right)\right)+\psi\left(X, \mu\left(U_{i, 2}\right)\right) \\
& \leqslant \frac{\psi\left(X, \varepsilon_{i}\right)}{4}+\frac{\psi\left(X, \varepsilon_{i}\right)}{4} \leqslant \frac{\psi\left(X, \varepsilon_{i}\right)}{2} .
\end{aligned}
$$

Если же точки $\left(x_{1}, y\right),\left(x_{2}, y\right) \in I^{2} \backslash\left(U_{i, 1} \cup U_{i, 2}\right)$, то выполнено неравенство

$$
\left|g_{i}\left(x_{1}, y\right)-g_{i}\left(x_{2}, y\right)\right| \leqslant \alpha_{i}\left|x_{1}-x_{2}\right| .
$$


Если же точки $\left(x, y_{1}\right),\left(x, y_{2}\right) \in I^{2} \backslash\left(U_{i, 1} \cup U_{i, 2}\right)$, то вьполнено неравенство

$$
\left|g_{i}\left(x, y_{1}\right)-g_{i}\left(x, y_{2}\right)\right| \leqslant \beta_{i}\left|y_{1}-y_{2}\right|
$$

Далее, для функций $g_{i}(x, y)$ введем множества $O_{i}=\left\{(t, s):\left|g_{i}(t, s)\right|>\gamma_{i}\right\}$. Тогда из (1), (14) и (11) имеем

$$
\begin{aligned}
\psi\left(X, \mu\left(O_{i}\right)\right) & \leqslant \frac{1}{\gamma_{i}}\left\|g_{i}(x, y) \mid X\right\| \\
& \leqslant \frac{1}{\gamma_{i}}\left\{\omega_{1}\left(h_{i-1}, f, X\right)+\omega_{1}\left(h_{i}, f, X\right)+\omega_{2}\left(\delta_{i-1}, f, X\right)+\omega_{2}\left(\delta_{i}, f, X\right)\right\} \\
& \leqslant \frac{\psi\left(X, \varepsilon_{i}\right)}{2} .
\end{aligned}
$$

Если же точки $\left(x_{1}, y\right),\left(x_{2}, y\right) \in I^{2} \backslash O_{i}$ или $\left(x, y_{1}\right),\left(x, y_{2}\right) \in I^{2} \backslash O_{i}$, то вьполнены неравенства

$$
\left|g_{i}\left(x_{1}, y\right)-g_{i}\left(x_{2}, y\right)\right| \leqslant \gamma_{i}, \quad\left|g_{i}\left(x, y_{1}\right)-g_{i}\left(x, y_{2}\right)\right| \leqslant \gamma_{i}
$$

Положим $W_{i}=U_{i, 1} \cup U_{i, 2} \cup O_{i}$. Тогда из неравенств (15), (17) получим

$$
\psi\left(X, \mu\left(W_{i}\right)\right) \leqslant \psi\left(X, \mu\left(O_{i}\right)\right)+\psi\left(X, \mu\left(U_{i, 1} \cup U_{i, 2}\right)\right) \leqslant \psi\left(X, \varepsilon_{i}\right) .
$$

Откуда из строгой монотонности $\psi(X, t)$ следует, что

$$
\mu\left(W_{i}\right) \leqslant \varepsilon_{i}
$$

Положим $W(\varepsilon)=\bigcup_{i=1}^{\infty} W_{i}$. Тогда из (5) и (19) следует, что вьполнено (8). Если теперь $\left(x_{1}, y\right),\left(x_{2}, y\right) \in I^{2} \backslash W(\varepsilon) \subseteq I^{2} \backslash\left(U_{i, 1} \cup U_{i, 2} \cup O_{i}\right)$, то для любого $i$ вьполнены неравенства (16), (18). Поэтому для каждого $k \in \mathbb{N}$ можно написать неравенство

$$
\begin{aligned}
\left|f\left(x_{1}, y\right)-f\left(x_{2}, y\right)\right| \leqslant & \left|f\left(h_{0}, \delta_{0} ; x_{1}, y\right)-f\left(h_{0}, \delta_{0} ; x_{2}, y\right)\right| \\
& +\sum_{i=1}^{k}\left|g_{i}\left(x_{1}, y\right)-g_{i}\left(x_{2}, y\right)\right|+\sum_{i=k+1}^{\infty}\left|g_{i}\left(x_{1}, y\right)-g_{i}\left(x_{2}, y\right)\right| \\
\leqslant & \left|f\left(h_{0}, \delta_{0} ; x_{1}, y\right)-f\left(h_{0}, \delta_{0} ; x_{2}, y\right)\right|+\left|x_{1}-x_{2}\right| \sum_{i=1}^{k} \alpha_{i}+2 \sum_{i=k+1}^{\infty} \gamma_{i} .
\end{aligned}
$$

Из соотношений (11), (12) получим

$$
\begin{aligned}
& \left|f\left(x_{1}, y\right)-f\left(x_{2}, y\right)\right| \leqslant\left|f\left(h_{0}, \delta_{0} ; x_{1}, y\right)-f\left(h_{0}, \delta_{0} ; x_{2}, y\right)\right| \\
& \quad+8 c_{0}\left|x_{1}-x_{2}\right| \sum_{i=1}^{k} \frac{1}{\psi\left(X, \varepsilon_{i}\right)}\left\{\frac{\omega_{1}\left(h_{i}, f, X\right)}{h_{i}}+\frac{\omega_{1}\left(h_{i-1}, f, X\right)}{h_{i-1}}\right\} \\
& \quad+4 \sum_{i=k+1}^{\infty} \frac{1}{\psi\left(X, \varepsilon_{i}\right)}\left\{\omega_{1}\left(h_{i}, f, X\right)+\omega_{1}\left(h_{i-1}, f, X\right)+\omega_{2}\left(\delta_{i}, f, X\right)+\omega_{2}\left(\delta_{i-1}, f, X\right)\right\} .
\end{aligned}
$$


Поскольку $\omega_{1}(h, f, X)$ и $\omega_{2}(\delta, f, X)$ - вогнутые функции, то они удовлетворяют неравенствам

$$
\begin{gathered}
\omega_{1}\left(h_{i}, f, X\right) \leqslant \omega_{1}\left(h_{i-1}, f, X\right), \quad \omega_{2}\left(\delta_{i}, f, X\right) \leqslant \omega_{2}\left(\delta_{i-1}, f, X\right), \\
\frac{\omega_{1}\left(h_{i-1}, f, X\right)}{h_{i-1}} \leqslant \frac{\omega_{1}\left(h_{i}, f, X\right)}{h_{i}} .
\end{gathered}
$$

Поэтому

$$
\begin{aligned}
\left|f\left(x_{1}, y\right)-f\left(x_{2}, y\right)\right| \leqslant & \left|f\left(h_{0}, \delta_{0} ; x_{1}, y\right)-f\left(h_{0}, \delta_{0} ; x_{2}, y\right)\right| \\
& +16 c_{0}\left|x_{1}-x_{2}\right| \sum_{i=1}^{k} \frac{1}{\psi\left(X, \varepsilon_{i}\right)} \frac{\omega_{1}\left(h_{i}, f, X\right)}{h_{i}} \\
& +8 \sum_{i=k+1}^{\infty} \frac{1}{\psi\left(X, \varepsilon_{i}\right)}\left\{\omega_{1}\left(h_{i-1}, f, X\right)+\omega_{2}\left(\delta_{i-1}, f, X\right)\right\},
\end{aligned}
$$

и можно положить $c_{1}=\max \left\{16 c_{0}, 8\right\}$.

Итак, неравенство (9) доказано. Если теперь $\left(x, y_{1}\right),\left(x, y_{2}\right) \in I^{2} \backslash W(\varepsilon)$, то неравенство (10) доказьвается аналогично.

Теорема доказана.

Обсудим теперь возможности описания "наилучших" частных модулей непрерьвности $\Omega_{1}(h, f, X), \Omega_{2}(\delta, f, X)$ в $(7)$, причем желательно “минимизировать" $\Omega_{1}(h, f, X)$, $\Omega_{2}(\delta, f, X)$ по последовательностям $\left\{h_{i}\right\},\left\{\delta_{i}\right\},\left\{\varepsilon_{i}\right\}$.

Лемма 1. Пусть $\omega(h)$ - некоторый вогнутый модуль непрерывности, $\omega(0)=0$, и точки $\left\{h_{i}\right\}$ выбраны так, что $\omega\left(h_{i}\right)=2^{-i}$. Если при некотором $q<1$ выполнено соотношение

$$
\varlimsup_{i \rightarrow \infty} \frac{\omega^{-1}\left(2^{-i-1}\right)}{\omega^{-1}\left(2^{-i}\right)}<\frac{q}{2}
$$

то существует $i_{0} \in \mathbb{N}$, начиная с которого выполняется неравенство

$$
\frac{\omega\left(h_{i}\right)}{h_{i}}<\frac{1+q}{2} \frac{\omega\left(h_{i+1}\right)}{h_{i+1}} .
$$

ДоКАЗАТЕЛЬСТВО. Поскольку $\omega\left(h_{i}\right)=2^{-i}$, то $h_{i}=\omega^{-1}\left(2^{-i}\right)$. Поэтому

$$
\frac{\omega\left(h_{i}\right)}{h_{i}}: \frac{\omega\left(h_{i+1}\right)}{h_{i+1}}=\frac{\omega\left(h_{i}\right)}{h_{i}} \frac{h_{i+1}}{\omega\left(h_{i+1}\right)}=\frac{2^{-i}}{\omega^{-1}\left(2^{-i}\right)} \frac{\omega^{-1}\left(2^{-i-1}\right)}{2^{-i-1}}=2 \frac{\omega^{-1}\left(2^{-i-1}\right)}{\omega^{-1}\left(2^{-i}\right)}
$$

Из последнего равенства и из (20) и следует утверждение леммы.

Лемма доказана. 
ТЕОРема 2. Пусть $X$ - симметричное пространство, фундаментальная функиия которого $\psi(X, t)$ строго монотонна. Пусть задана непрерывная функиия $f(x, y)$ с заданными частными модулями непрерывности $\omega_{1}(h)=\omega_{1}(h, f, X) u$ $\omega_{2}(\delta)=\omega_{2}(\delta, f, X)$. Пусть последовательности $\left\{h_{i}\right\},\left\{\delta_{i}\right\}$ определены из соотношений $\omega_{1}\left(h_{i}\right)=2^{-i}, \omega_{2}\left(\delta_{i}\right)=2^{-i}$ и при некотором $q \in(0,1)$ выполнены соотноиения

$$
\varlimsup_{i \rightarrow \infty} \frac{\omega_{1}^{-1}\left(2^{-i-1}\right)}{\omega_{1}^{-1}\left(2^{-i}\right)}<\frac{q}{2}, \quad \varlimsup_{i \rightarrow \infty} \frac{\omega_{2}^{-1}\left(2^{-i-1}\right)}{\omega_{2}^{-1}\left(2^{-i}\right)}<\frac{q}{2} .
$$

Пусть задано $\varepsilon>0$ и последовательность $\left\{\varepsilon_{i}\right\}$ удовлетворяет (5) $и$

$$
\lim _{i \rightarrow \infty} \frac{\varepsilon_{i}}{\varepsilon_{i+1}}=1 \text {. }
$$

Тогда существует мнохество $W(\varepsilon)$ такое, что выполнены соотношения:

a) $\mu(W(\varepsilon))<\varepsilon$

б) если $\left(x_{1}, y\right),\left(x_{2}, y\right) \in I^{2} \backslash W(\varepsilon) u\left|x_{1}-x_{2}\right| \in\left[h_{j}, h_{j-1}\right)$, mo

$$
\left|f\left(x_{1}, y\right)-f\left(x_{2}, y\right)\right| \leqslant\left|f\left(h_{0}, \delta_{0} ; x_{1}, y\right)-f\left(h_{0}, \delta_{0} ; x_{2}, y\right)\right|+c \frac{\omega_{1}\left(\left|x_{1}-x_{2}\right|\right)}{\psi\left(X, \varepsilon_{j}\right)}
$$

в) $е с л u\left(x, y_{1}\right),\left(x, y_{2}\right) \in I^{2} \backslash W(\varepsilon) u\left|y_{1}-y_{2}\right| \in\left[\delta_{j}, \delta_{j-1}\right)$, mo

$$
\left|f\left(x, y_{1}\right)-f\left(x, y_{2}\right)\right| \leqslant\left|f\left(h_{0}, \delta_{0} ; x, y_{1}\right)-f\left(h_{0}, \delta_{0} ; x, y_{2}\right)\right|+c \frac{\omega_{2}\left(\left|y_{1}-y_{2}\right|\right)}{\psi\left(X, \varepsilon_{j}\right)}
$$

Здесь с не зависит от $i \in \mathbb{N}$.

ДокАЗАТЕЛЬСТво. Из определения последовательностей $\left\{h_{i}\right\},\left\{\delta_{i}\right\}$ и из (21) следует, что выполнено (6). Поэтому для доказательства теоремы 2 согласно теореме 1 достаточно оценить функции $\Omega_{1}(h, f, X), \Omega_{2}(\delta, f, X)$. Оценим функцию $\Omega_{1}(h)=\Omega_{1}(h, f, X)$. Из $(22)$ и квазивогнутости фундаментальной функции $\psi(X, t)$ следует равенство

$$
\lim _{i \rightarrow \infty} \frac{\psi\left(X, \varepsilon_{i}\right)}{\psi\left(X, \varepsilon_{i+1}\right)}=1
$$

Из (21), леммы 1 и последнего равенства следует, что при некотором $q_{1} \in(0,1)$ при всех достаточно больших $i$ вьполняются неравенства

$$
\frac{\omega_{1}\left(h_{i+1}\right)}{\psi\left(X, \varepsilon_{i+1}\right)} \leqslant q_{1} \frac{\omega_{1}\left(h_{i}\right)}{\psi\left(X, \varepsilon_{i}\right)}, \quad \frac{\omega_{1}\left(h_{i}\right)}{h_{i} \psi\left(X, \varepsilon_{i}\right)} \leqslant q_{1} \frac{\omega_{1}\left(h_{i+1}\right)}{h_{i+1} \psi\left(X, \varepsilon_{i+1}\right)} .
$$

Следовательно, найдется константа $c_{2}$ такая, что при всех $k \in \mathbb{N}$ выполняются неравенства

$$
\begin{aligned}
\sum_{i=1}^{k} \frac{\omega_{1}\left(h_{i}\right)}{h_{i} \psi\left(X, \varepsilon_{i}\right)} & \leqslant c_{2} \frac{\omega_{1}\left(h_{k}\right)}{h_{k} \psi\left(X, \varepsilon_{k}\right)}, \\
\sum_{i=k+1}^{\infty} \frac{\omega_{1}\left(h_{i-1}\right)+\omega_{2}\left(\delta_{i-1}\right)}{\psi\left(X, \varepsilon_{i}\right)} & \leqslant c_{2} \frac{\omega_{1}\left(h_{k}\right)}{\psi\left(X, \varepsilon_{k+1}\right)} .
\end{aligned}
$$


Поэтому если $\left(x_{1}, y\right),\left(x_{2}, y\right) \in I^{2} \backslash W(\varepsilon)$ и $\left|x_{1}-x_{2}\right| \in\left[h_{j}, h_{j-1}\right)$, то из $(25),(26)$ получим

$$
\begin{aligned}
\Omega_{1}\left(\left|x_{1}-x_{2}\right|\right) & \leqslant\left|x_{1}-x_{2}\right| \sum_{i=1}^{j-1} \frac{\omega_{1}\left(h_{i}\right)}{h_{i} \psi\left(X, \varepsilon_{i}\right)}+\sum_{i=j}^{\infty} \frac{\omega_{1}\left(h_{i-1}\right)+\omega_{2}\left(\delta_{i-1}\right)}{\psi\left(X, \varepsilon_{i}\right)} \\
& \leqslant c_{2}\left\{\left|x_{1}-x_{2}\right| \frac{\omega_{1}\left(h_{j-1}\right)}{h_{j-1} \psi\left(X, \varepsilon_{j-1}\right)}+\frac{2 \omega_{1}\left(h_{j}\right)}{\psi\left(X, \varepsilon_{j}\right)}\right\} \\
& \leqslant c_{2}\left\{\frac{2 \omega_{1}\left(h_{j}\right)}{\psi\left(X, \varepsilon_{j-1}\right)}+\frac{2 \omega_{1}\left(h_{j}\right)}{\psi\left(X, \varepsilon_{j}\right)}\right\} \\
& \leqslant c_{2}\left\{\frac{2 \omega_{1}\left(\left|x_{1}-x_{2}\right|\right)}{\psi\left(X, \varepsilon_{j}\right)} \frac{\psi\left(X, \varepsilon_{j}\right)}{\psi\left(X, \varepsilon_{j-1}\right)}+\frac{2 \omega_{1}\left(\left|x_{1}-x_{2}\right|\right)}{\psi\left(X, \varepsilon_{j}\right)}\right\} \\
& \leqslant c_{2}\left\{c_{3} \frac{2 \omega_{1}\left(\left|x_{1}-x_{2}\right|\right)}{\psi\left(X, \varepsilon_{j}\right)}+\frac{2 \omega_{1}\left(\left|x_{1}-x_{2}\right|\right)}{\psi\left(X, \varepsilon_{j}\right)}\right\} \leqslant c \frac{\omega_{1}\left(\left|x_{1}-x_{2}\right|\right)}{\psi\left(X, \varepsilon_{j}\right)}
\end{aligned}
$$

Соотношение (23) доказано; аналогично проверяется и (24).

Теорема доказана.

Теперь мы сформулируем теорему, показывающую неусиляемость теоремы 2.

Теорема 3. Пусть $X$ - симметричное пространство, для фундаментальной функиии которого выполнено условие

$$
\lim _{t \rightarrow 0} \psi(X, t)=0 .
$$

Пусть $\omega_{1}(h)$ и $\omega_{2}(\delta)$ - два модуля непрерывности, для которых выполнено (21). Пусть для последовательности $\left\{b_{i}\right\}$ выполнены соотношения

$$
b_{i} \geqslant b_{i+1}, \quad \lim _{i \rightarrow \infty} \frac{b_{i}}{b_{i+1}}=1, \quad \sum_{i=1}^{\infty} b_{i}=\infty
$$

Тогда найдется непрерьвная функиия $f \in X$ такая, что $\omega_{1}(h, f, X) \leqslant c \omega_{1}(h)$, $\omega_{2}(\delta, f, X) \leqslant c \omega_{2}(\delta)$ и для любого множсества $D$ с $\mu(D)>0$ выполнены соотношения

$$
\begin{gathered}
\varlimsup_{i \rightarrow \infty} \frac{\psi\left(X, b_{i}\right)}{\omega_{1}\left(2^{-i}\right)} \sup \left\{\left|f\left(x_{1}, y\right)-f\left(x_{2}, y\right)\right|:\left(x_{1}, y\right),\left(x_{2}, y\right) \in D,\left|x_{1}-x_{2}\right|<2^{-i}\right\}>0, \\
\varlimsup_{i \rightarrow \infty} \frac{\psi\left(X, b_{i}\right)}{\omega_{2}\left(2^{-i}\right)} \sup \left\{\left|f\left(x, y_{1}\right)-f\left(x, y_{2}\right)\right|:\left(x, y_{1}\right),\left(x, y_{2}\right) \in D,\left|y_{1}-y_{2}\right|<2^{-i}\right\}>0 .
\end{gathered}
$$

ДокаЗАТЕЛЬСТво. По симметричному пространству $X$ на единичном квадрате построим симметричноепространство $X_{0}$ на единичном отрезке, полагая $\left\|g\left|X_{0}\|=\| \bar{g}\right| X\right\|$, где $\bar{g}(x, y) \equiv g(x)$. Очевидно, что фундаментальные функции $X$ и $X_{0}$ совпадают. Согласно теореме 4 работы [2] построим две непрерьвные одномерные функции $f_{1}(x), f_{2}(x)$, для которых выполнены условия

$$
\omega\left(h, f_{1}, X_{0}\right) \leqslant \omega_{1}(h), \quad \omega\left(h, f_{2}, X_{0}\right) \leqslant \omega_{2}(h) ;
$$


для любого множества $M \subset(0,1)$ с $\mu(M)>0$ вьполнены соотношения

$$
\begin{gathered}
\varlimsup_{i \rightarrow \infty} \frac{\psi\left(X, b_{i}\right)}{\omega_{1}\left(2^{-i}\right)} \sup \left\{\left|f_{1}\left(x_{1}\right)-f_{1}\left(x_{2}\right)\right|: x_{1}, x_{2} \in M,\left|x_{1}-x_{2}\right|<2^{-i}\right\}>0 \\
\varlimsup_{i \rightarrow \infty} \frac{\psi\left(X, b_{i}\right)}{\omega_{2}\left(2^{-i}\right)} \sup \left\{\left|f_{2}\left(y_{1}\right)-f_{2}\left(y_{2}\right)\right|: y_{1}, y_{2} \in M,\left|y_{1}-y_{2}\right|<2^{-i}\right\}>0 .
\end{gathered}
$$

Для окончания доказательства теоремы достаточно положить $f(x, y)=f_{1}(x) f_{2}(y)$ и использовать известную связь меры плоского множества с мерами его сечений (см., например, [9, с. 318]).

Теорема доказана.

Проиллюстрируем приведенные теоремы примером.

ПРимеР. Пусть задана функция $f: I^{2} \rightarrow \mathbb{R}$, для которой $\omega_{1}(h, f, X) \leqslant c_{1} h^{\alpha}$ с $\alpha \in$ $(0,1), \omega_{2}(\delta, f, X) \leqslant c_{1} \delta^{\beta} \mathrm{c} \beta \in(0,1)$.

Если положить $\varepsilon_{i}=c_{2} i^{-(1+\sigma)}$ c $\sigma>0$, то из теорем 1,2 следует, что найдется множество меры больше $1-\varepsilon$, на котором равномерные частные модули непрерывности допускают оценки

$$
\begin{aligned}
& \omega_{1}\left(h, f, L^{\infty}\right) \leqslant c_{3} \frac{h^{\alpha}}{\psi\left(X,\left(\log \frac{1}{h}\right)^{-(1+\sigma)}\right)}, \\
& \omega_{2}\left(\delta, f, L^{\infty}\right) \leqslant c_{3} \frac{\delta^{\beta}}{\psi\left(X,\left(\log \frac{1}{\delta}\right)^{-(1+\sigma)}\right)} .
\end{aligned}
$$

Если же положить $\varepsilon_{i}=c_{2} i^{-1}(\log i)^{-(1+\sigma)}$ с $\sigma>0$, то из теорем 1,2 следует, что найдется множество меры больше $1-\varepsilon$, на котором равномерные частные модули непрерьвности допускают оценки

$$
\begin{aligned}
& \omega_{1}\left(h, f, L^{\infty}\right) \leqslant c_{3} \frac{h^{\alpha}}{\psi\left(X,\left(\log \frac{1}{h}\right)^{-1}\left(\log \log \frac{1}{h}\right)^{-(1+\sigma)}\right)} \\
& \omega_{2}\left(\delta, f, L^{\infty}\right) \leqslant c_{3} \frac{\delta^{\beta}}{\psi\left(X,\left(\log \frac{1}{\delta}\right)^{-1}\left(\log \log \frac{1}{\delta}\right)^{-(1+\sigma)}\right)}
\end{aligned}
$$

и т.д.

$\mathrm{C}$ другой стороны, если положить $b_{i}=i^{-1}$, то из теоремы 3 следует, что найдется непрерьвная $f$ с $\omega_{1}(h, f, X) \leqslant c_{1} h^{\alpha}, \omega_{2}(\delta, f, X) \leqslant c_{1} \delta^{\beta}$, для которой частные равномерные модули непрерьвности, вычисленные по любому множеству положительной меры, допускают оценки

$$
\begin{gathered}
\varlimsup_{h \rightarrow 0} \frac{\omega_{1}\left(h, f, L^{\infty}\right)}{h^{\alpha}} \psi\left(X,\left(\log \frac{1}{h}\right)^{-1}\right)>0, \\
\varlimsup_{\delta \rightarrow 0} \frac{\omega_{2}\left(\delta, f, L^{\infty}\right)}{\delta^{\alpha}} \psi\left(X,\left(\log \frac{1}{\delta}\right)^{-1}\right)>0 .
\end{gathered}
$$

Если же положить $b_{i}=(i \log i)^{-1}$ и применить теорему 3 , то найдется непрерьвная $f$ с $\omega_{1}(h, f, X) \leqslant c_{1} h^{\alpha}, \omega_{2}(\delta, f, X) \leqslant c_{1} \delta^{\beta}$, для которой частные равномерные модули 
непрерьвности, вычисленные по любому множеству положительной меры, допускают оценки

$$
\begin{gathered}
\varlimsup_{h \rightarrow 0} \frac{\omega_{1}\left(h, f, L^{\infty}\right)}{h^{\alpha}} \psi\left(X,\left(\log \frac{1}{h}\right)^{-1}\left(\log \log \frac{1}{h}\right)^{-1}\right)>0, \\
\varlimsup_{\delta \rightarrow 0} \frac{\omega_{2}\left(\delta, f, L^{\infty}\right)}{\delta^{\alpha}} \psi\left(X,\left(\log \frac{1}{\delta}\right)^{-1}\left(\log \log \frac{1}{\delta}\right)^{-1}\right)>0 .
\end{gathered}
$$

Таким образом, “наилучшей” оценки модуля непрерывности в рассматриваемой ситуации нет.

\section{СПИСОК ЦИТИРОВАННОЙ ЛИТЕРАТУРЫ}

[1] Зигмунд А. Тригонометрические ряды. Т. 1, 2. М.: Мир, 1965.

[2] Бережной Е.И. Оценки равномерного модуля непрерывности функций из симметричньх пространств // Изв. РАН. 1996. Т. 60. № 2. С. 3-20.

[3] Брудный Ю. А. Пространства, определяемые с помощью локальных приближений // Тр. MMO. 1971. T. 24. C. $69-132$.

[4] Коляда В. И. Оценки максимальных функций, связанных с локальной гладкостью // Докл. AH CCCP. 1987. T. 293. C. 534-537.

[5] Осколков К.И. Равномерный модуль непрерывности суммируемьх функций на множествах положительной меры // Докл. АН СССР. 1976. Т. 229. № 2. С. 304-306.

[6] Ульянов П. Л. О работах Н.Н. Лузина по метрической теории функций // УМН. 1985. T. 40. № 3. C. 15-70.

[7] Крейн С. Г., Петунин Ю. И., Семёнов Е. М. Интерполяция линейных операторов. М.: Наука, 1977.

[8] Дзядык В.К. Введение в теорию равномерного приближения функций полиномами. М.: Наука, 1977.

[9] Натансон И. П. Теория функций вещественной переменной. М.: Наука, 1974.

Ярославский государственньй университет им. П. Г. Демидова 